\title{
Multiplexed Model Predictive Control of Interconnected Systems
}

\author{
Tri $\operatorname{Tran}^{a}$, D. Zhou ${ }^{b}$, K-V. Ling ${ }^{b}$, and Jan M. Maciejowski ${ }^{c}$
}

\begin{abstract}
A Multiplexed Model Predictive Control (MMPC) scheme with Quadratic Dissipativity Constraint (QDC) for interconnected systems is presented in this paper. A centralized MMPC is designed for the global system, wherein the controls of subsystems are updated sequentially to reduce the computational time. In MMPC, the global state vector of the interconnected system is required by the optimization. The QDC is converted into an enforced stability constraint for the MMPC as an alternative to the terminal constraint and terminal cost in this approach. The nominal recursive feasibility for the global system and the iterative feasibility for the local subsystems are obtained via set operations on the invariant sets. The admissible sets for the control inputs are obtained and employed in this approach for the QDC-based stability constraint. The set operations are speed up by multiple magnitudes thanks to the implementation of multiplexed inputs in MMPC. Numerical simulations with Automatic Generation Control (AGC) in power systems having tie-lines demonstrate the theoretical development.
\end{abstract}

\section{INTRODUCTION}

Multiplexed Model Predictive Control (MMPC) was initially introduced to facilitate fast and distributed applications [1]. The computational complexity of a traditional MPC problem tends to vary as $O\left(\left(m \times N_{u}\right)^{3}\right)$ [2], [3], where $m$ is the number of control inputs, and $N_{u}$ is the horizon length. If a given MPC problem has the smallest possible update interval of $T$ seconds, MMPC will update "only one control variable at a time, to exploit the reduced complexity, and to update successive inputs at intervals smaller than $T$, typically $T / m$ " [1]. There are thus $m$ updates within each updating interval $T$. The distinct feature of MMPC rests on the sequential updates for the control input vector, which sets it apart from a decentralized MPC in a similar manner to the differences between the Jacobi and Gauss-Seidel iterative algorithms for inverting a matrix [4], as indicated in [1]. MMPCs have been successfully implemented in FPGA and applied to a variety of problems such as thermal processing in semiconductor manufacturing [5], vehicle suspension control [6], jet engine control [7], and airplane control [8]. An

\footnotetext{
${ }^{a}$ Tri Tran is currently with Cambridge CARES, Nanyang Technological University, 62 Nanyang Drive, Singapore 637459. Email: tctran@ntu.edu.sg, tctran@ieee.org

${ }^{b}$ D. Zhou and K-V Ling are with the School of Electrical and Electronic Engineering, Nanyang Technological University, Block S2, 50 Nanyang Ave, Singapore 639798. Email: ekvlingentu. edu.sg

${ }^{c}$ Jan M. Maciejowski is with Department of Engineering, University of Cambridge, Cambridge CB2 1PZ, United Kingdom, and with the Energy Research Institute at Nanyang Technological University, ERI@N. Email: jmm@eng. cam.ac.uk

The authors acknowledge the support by the Singapore National Research Foundation (NRF) under its Campus for Research Excellence And Technological Enterprise (CREATE) programme and the Cambridge Centre for Advanced Research in Energy Efficiency in Singapore (Cambridge CARES), C4T project.
}

extension of MMPC called channel-hopping MMPC can be found in [9].

In this paper, the Quadratic Dissipativity Constraint (QDC), introduced previously in [10], is applied to the MMPC of interconnected systems, as an alternative to terminal cost and terminal state constraint. The complexity of online computations for the QDC in MMPC is reduced by using multiplexed inputs which have smaller vector sizes than the entire control vector. Furthermore, The QDC leads to a constraint on the current-time control only, rather than over the control horizon. This further reduces the computational complexity. We will show in Section IV-B that the so-called local one-step admissible control set is an invariant set, and its corresponding invariant set with respect to predictive states belongs to the family of dynamic terminal constraint sets [11] for a local subsystem taking inter-subsystem interactions into account. The smaller size multiplexed inputs in MMPC will also help reduce the computation for a local one-step admissible control set.

Figure 1 depicts the position of MMPC in a typical interconnected system, in which there are $m$ subsystems $\mathcal{S}_{i}$, $i=1 \ldots m$, physically interconnected in a fixed connection topology. The MMPC computes the control vector $u_{i}$ for each subsystem $\mathcal{S}_{i}$ at every time step. The computation can be distributed to the $m$ subsystems, provided that the subcontrollers can communicate with each other. The complete input vector $\left[u_{1}^{T}, \ldots, u_{m}^{T}\right]^{T}$ is thus updated sequentially in $m$ time steps. The whole process is periodic, with period $m$ time steps.

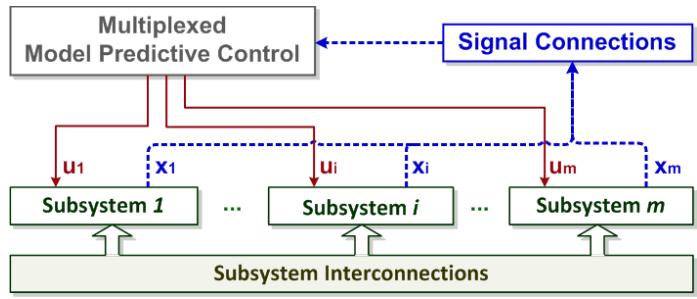

Fig. 1. Block diagram of an interconnected system with MMPC.

Within this set up, the state vectors of subsystems are collected by the MMPC via communication links. We assume that the data sent through the communication channels are perfect without sporadic data losses or delays. The question of robustness to data losses is the subject of other work.

In this paper, MMPC is formulated without a specially chosen terminal cost or terminal constraint, which were used in previous developments of MMPC ([1], [2], [8]), but replaces them with the QDC. For the recursive feasibility of 
MMPC, invariant sets [12] and set operations (of Minkowski sum and Pontryagin difference) will be used in this work. These set operations make use of the available information data of state and interaction vectors at every time step. The one-step admissible control set is derived from the maximal output admissible set [13], before being combined with the QDC-based stability constraint for MMPC. The recursive feasibility in the face of hard constraints is thus guaranteed with the one-step admissible control sets for both the global system and local subsystems.

The rest of the paper is organized as follows. The system and control models are provided in Section 2. The QDCbased stability constraint and the traditional MPC are also presented in this section. The MMPC optimization problem is outlined in Section 3. We show in this section that the QDC-based stability constraint is easily fit to the MMPC optimization. Section 4 is reserved for the recursive feasibility condition that is derived from the one-step admissible multiplexed-control sets. The stability condition for constrained systems is also presented in this section. An illustrative example is given in Section 5. Section 6 concludes this paper.

\section{PRELIMINARIES}

\section{A. Notation}

Upper and lower case letters denote matrices and column vectors, respectively. (. $)^{T}$ denotes the transpose. $\|u\|_{2}$ is the $\ell_{2}$-norm of vector $u$. $\|x\|_{\mathcal{Q}}^{2}$ is $x^{T} \mathcal{Q} x, \mathcal{Q} \succcurlyeq 0$. In the discrete time domain, the time index is denoted by $k, k \in \mathbb{Z}$.

\section{B. System model}

Consider an interconnected system $\Sigma$ consisting of $m$ subsystems, each denoted as $\mathcal{S}_{i}, i=1, \ldots, m$ and has a discrete-time state space model of the form:

$$
\mathcal{S}_{i}:\left\{\begin{array}{l}
x_{i}(k+1)=A_{i} x_{i}(k)+B_{i} u_{i}(k)+E_{i} v_{i}(k), \\
w_{i}(k)=F_{i} x_{i}(k)
\end{array}\right.
$$

where $x_{i}(k) \in \mathbb{R}^{n_{i}}, u_{i}(k) \in \mathbb{R}^{h_{i}}, v_{i}(k) \in \mathbb{R}^{p_{i}}$ and $w_{i}(k) \in \mathbb{R}^{q_{i}}$ are the state, control input, interactive input and interactive output vectors, respectively. The constraints $\left\|u_{i}(k)\right\|_{2}^{2} \leq \eta_{i},\left\|x_{i}(k)\right\|_{2}^{2} \leq \rho_{i}$, and $\left\|v_{i}(k)\right\|_{2}^{2} \leq \theta_{i}$ are considered.

The input $v_{i}(k)$ of subsystem $\mathcal{S}_{i}$ and the output $w_{j}(k)$ of subsystem $\mathcal{S}_{j}, i \neq j$, are connected to each other in an arbitrary topology. Using the definitions of block-diagonal matrices $A_{L}:=\operatorname{diag}\left[A_{i}\right]_{1}^{m}, x_{L}:=\left[x_{1}^{T} \ldots x_{m}^{T}\right]^{T}$, and similarly for other matrices, the large-scale system $\Sigma$ is obtained in the following:

$$
\Sigma:\left\{\begin{array}{l}
x_{L}(k+1)=\left(A_{L}+E_{L} H F_{L}\right) x_{L}(k)+B_{L} u(k), \\
v(k)=H w(k),
\end{array}\right.
$$

with a global coupling matrix $H$ of entries 1 or 0 only.

The following augmented model will be used in the MMPC optimization:

$$
x(k+1)=A x(k)+B \triangle u(k),
$$

where $x(k):=\left[\begin{array}{c}x_{L}(k) \\ u(k-1)\end{array}\right], A:=\left[\begin{array}{cc}A_{\Sigma} & B_{L} \\ 0 & I\end{array}\right], B:=\left[\begin{array}{c}B_{L} \\ I\end{array}\right]$, in which $A_{\Sigma}:=A_{L}+E_{L} H F_{L}, x \in \mathbb{X}=\mathbb{X}_{1} \times \ldots \times \mathbb{X}_{m}$, and $\triangle u_{i}(k):=u_{i}(k)-u_{i}(k-1)$.

Assumption 1: $\left(A_{i}, B_{i}\right), i=1 \ldots m$, and $(A, B)$ are controllable.

\section{Quadratic dissipativity constraint}

As in [10], [14], the QDC-based stability constraint is

$$
\triangle u(k)^{T} R \triangle u(k)+2 x(k)^{T} S \triangle u(k)+\psi(k) \leq 0,
$$

where $\quad \psi(k):=x(k)^{T} Q x(k)-\beta \xi(k-1), \quad 0<\beta<1$, and $\xi(k)$ denotes the supply rate $\xi(x(k), u(k))$, defined as

$$
\xi(x(k), u(k)):=\left[x(k)^{T} \triangle u(k)^{T}\right]\left[\begin{array}{cc}
Q & S \\
S^{T} & R
\end{array}\right]\left[\begin{array}{c}
x(k) \\
\triangle u(k)
\end{array}\right] .
$$

The multiplier matrices $Q, S, R$ are to be determined from the stability and recursive feasibility conditions derived in Section IV. To achieve the closed-loop system stability, it also requires that the open-loop system $\Sigma$ is dissipative with the following dissipation inequality:

$$
V(x(k+1))-\alpha V(x(k)) \leq \xi(x(k), u(k)), 0<\alpha<1 .
$$

$V(x)=x^{T} P x, P \succ 0$ is considered in this work. The stability condition for unconstrained systems with QDC is stated below. The notion of input-and-power-to-state stabilizability (IpSS), defined in [10], is used in this sufficient stability condition.

Theorem 1: Let $\xi(0)>0$ and $0<\alpha<\beta<1$. Consider $\Sigma$ without control and state constraints. Given $x_{0}$, any $\triangle u(k)$ satisfying (4) which have $Q, S, R$ solve the following LMI optimization, asymptotically stabilizes $\Sigma$ in IpSS sense:

$$
\begin{gathered}
\min _{P, Q, S, R} x_{0}^{T} P x_{0} \\
\text { s.t. }\left[\begin{array}{ccc}
P & P A & P B \\
* & \alpha P+Q & S \\
& * & R
\end{array}\right] \succ 0, P \succ 0, Q \prec 0, R \succ 0 .
\end{gathered}
$$

Proof: Refer to [10], realizing that the main LMI is the dissipative condition for $\Sigma$, see, e.g. [15].

\section{Multiplexed Model Predictive Control With QUADRATIC DissipaTIVITY CONSTRAINT}

\section{A. Basics of $M M P C$}

In our context, at each time step $k$ MMPC optimizes the inputs of subsystem $\mathcal{S}_{\sigma(k)}$, where the indexing function $\sigma(k)=(k \bmod m)+1$ (i.e. the vector $\left.u_{i}, i=\sigma(k)\right)$, over a prediction horizon. At the next time step $k+1$, it optimizes the inputs of subsystem $\sigma(k+1)$. Since $\sigma(k)$ is periodic, with period $m$, all of the inputs will have been optimized after $m$ time steps, and the cycle of optimization re-commences. This is illustrated in Figure 2, in which the inputs being optimized are shown in bold font.

In each MMPC optimization, the global state vector $x(k)$ is used. Those elements of $u$ which are not being optimized, 


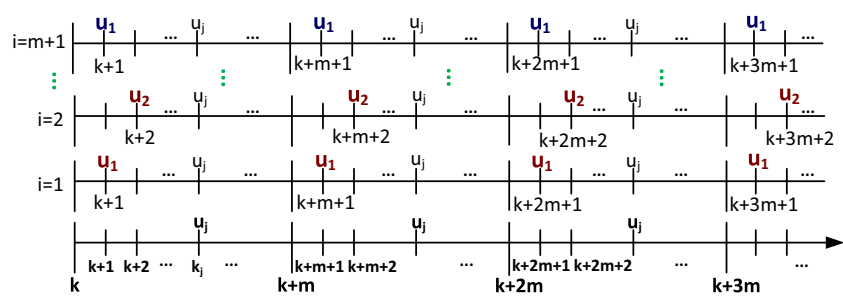

Fig. 2. Sequential control updates in MMPC with $m$ step cycle.

namely $u_{j}(k)$ with $j \in\{\sigma(k)+\nu m\}$ for positive integer $\nu$, are either known from past measurements, or are assumed to take the values most recently planned for them by other optimizations. For MMPC of interconnected systems, the state vector $x(k)$ of $\Sigma$ is known via the communication links between the local subsystems and the controller, while the multiplexed input $u_{i}$ is the control vector of a local subsystem $\mathcal{S}_{i}$. The patterns of control moves are illustrated in Figure 3.
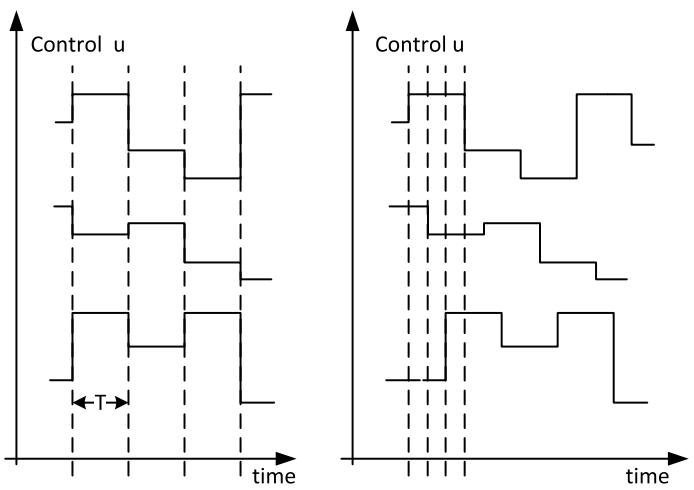

Fig. 3. Patterns of input moves for conventional MPC (left), and for MMPC (right).

Since the MMPC will update the $m$ input vectors of $\Sigma$ one at a time, (3) is recast for MMPC as follows:

$$
x(k+1)=A x(k)+\tilde{B}(k) \triangle \tilde{u}(k),
$$

where $\triangle \tilde{u}(k):=\triangle u_{\sigma(k)}(k), \tilde{B}(k):=B_{\sigma(k)}$.

In the following, the MMPC optimization is formulated without the terminal penalty function that is needed to satisfy the stability condition stated in Theorem 1, equation (6), in [2], neither the terminal constraint. The QDC-based stability constraint is employed instead to guarantee the stability of the closed-loop system.

\section{B. $M M P C$ algorithm with $Q D C$}

At each time step $k$, assume that all "planned" subsystem control vectors $\triangle \tilde{u}_{k+\ell \mid k-1}, \ell \neq 0, m, 2 m, \ldots$, are known to the MMPC. MMPC will only optimize over inputs to the subsystem about to be moved at each time step. MMPC solves the following finite-time constrained linear periodic control problem [2]:

$$
\min \mathcal{J}_{k}=\sum_{\ell=0}^{N-1}\left\|x_{k+\ell \mid k}^{T}\right\|_{\mathcal{X}}^{2}+\left\|\Delta \tilde{u}_{k+\ell \mid k}^{T}\right\|_{\mathcal{U}}^{2},
$$

$$
\begin{array}{r}
\text { w.r.t. } \triangle \tilde{u}_{k+\ell \mid k},(\ell=0, m, 2 m, \ldots, N-1) \\
\text { s.t. } \triangle \tilde{u}_{k+\ell \mid k}+u_{\sigma(k+\ell) \mid k-1} \in \mathbb{U}_{\sigma(k+\ell)},(\ell=0, m, \ldots, N-1) \\
x_{k+\ell \mid k} \in \mathbb{X},(\ell=1,2, \ldots, N-1) \\
x_{k+\ell+1 \mid k}=A x_{k+\ell \mid k}+B_{\sigma(k+\ell)} \triangle \tilde{u}_{k+\ell \mid k}, \quad(8 \mathrm{c}) \\
\triangle \tilde{u}_{k+\ell \mid k}=\triangle \tilde{u}_{k+\ell \mid k-1},(\ell \neq 0, m, 2 m, \ldots, N-1), \quad(8 \mathrm{f}) \\
\triangle \tilde{u}_{k \mid k}^{T} R \triangle \tilde{u}_{k \mid k}+2 x_{k \mid k}^{T} S \triangle \tilde{u}_{k \mid k}+\psi_{k} \leq 0,
\end{array}
$$

where $x_{k \mid k}=x(k)$ and $\psi_{k}, R, S$, are as defined in (4).

The MMPC procedure is provided below for clarity.

Algorithm 1 (MMPC with QDC-based stability constraint) Initialization: Set $k \equiv k_{0}$. Initialize $u(0)$ by solving Problem (8) over all decision variables $\triangle \tilde{u}_{k+\ell \mid k}, \ell=0,1, \ldots, N-1$, respecting only constraints $(8 \mathrm{c})-(8 \mathrm{e})$.

1) Apply control move $\triangle u_{\sigma(k), k}=\triangle \tilde{u}_{k \mid k}$.

2) Store planned moves $\triangle \tilde{u}_{k+j \mid k}, j=m, 2 m, \ldots, N_{u}$, where $N_{u}=(m-1) m$.

3) Pause for one time step, increment $k$, obtain new measurement $x_{k}$.

4) Solve problem (8), respecting all constraints in (8).

5) Go to step 1.

Remark 1: The stability constraint (8g) with dynamic multiplier matrices $Q, S, R$ will be updated at each time step $k$ using the LMIs derived in the stability and recursive feasibility conditions. Theses conditions ensure that the ellipsoidal constraint (8g) w.r.t. $\triangle \tilde{u}_{k \mid k}$ will intersect the ball of control constraint $\left\|u_{i}\right\| \leq \eta_{i}$ and satisfy the one-step admissible control condition derived in Section IV.

\section{RECURSIVE FEASIBILITY WITH INVARIANT SETS}

In the design of standard MPCs, the state trajectory beyond the predictive horizon should belong to a terminal constraint set, which is positively invariant set, denoted as $\mathbb{X}_{f},[16]$, to guarantee the recursive feasibility of MPC. For state feedback, the terminal constraint set is often chosen as the maximal output admissible set, $O_{\infty}$ [17] of the closed-loop system of the form $x(k+1)=\left(A+B K_{f}\right) x(k)$, where the control law $\triangle u=K_{f} x$ is the optimal controller for the unconstrained infinite horizon problem, which is the standard linear quadratic (LQ) problem. The state vector is the output vector herein. On the ground of the maximal output admissible set $\mathbb{X}_{f}$, the initial feasible set is then computed.

The set $\mathbb{X}_{\mathrm{r}}$ is the initial feasible set w.r.t $\mathbb{X}_{\mathrm{f}}$ for system $x(k+1)=A(k)+B \triangle u(k)$, with constraints $x \in \mathbb{X}, \triangle u \in$ $\mathbb{U}_{\triangle}[18]$, if and only if there exists an admissible control law that will drive the state of the system into $\mathbb{X}_{\mathrm{f}}$ in $N$ steps or less from $\mathbb{X}_{\mathrm{r}}$, while keeping the evolution of $x$ inside $\mathbb{X}$, i.e.

$$
\begin{aligned}
\mathbb{X}_{\mathrm{r}}:=\{x(k) & \in \mathbb{X} \mid \exists\left\{\triangle u(k) \in \mathbb{U}_{\triangle}\right\}_{k=0}^{N-1}: \\
& \left.\{x(k) \in \mathbb{X}\}_{k=0}^{N-1} \text { and } x(N) \in \mathbb{X}_{\mathrm{f}}\right\}
\end{aligned}
$$

The above initial feasible set $\mathbb{X}_{\mathrm{r}}$ is determined by computing backward from the terminal constraint set $\mathbb{X}_{f}$ using 
set operations. The representation of $\mathbb{X}_{r}$ versus $\mathbb{X}_{f}$ with Minkowski sum for discrete-time systems can be found in [19]. The constrained optimization of MPC is then recursively feasible (for all time $k \geq 0$ ) if and only if the initial state $x(0)$ belongs to the initial feasible set $\mathbb{X}_{\mathrm{r}}$.

The existing results presented in this section are applied to the determination of the recursive feasibility condition of an MMPC applying the QDC-based stability constraint. The nominally recursive feasibility condition stipulates the containment of the global one-step admissible control set inside the global initial feasible set.

\section{A. Global recursive feasibility}

The global one-step admissible control set is firstly defined. Denote $\mathbb{U}:=\mathbb{U}_{1} \times \ldots \times \mathbb{U}_{m}$.

Definition 1: Given the current state $x(k) \in \mathbb{X}_{\mathrm{r}}$ and the past control $u(k-1)$, the one-step admissible control set $\mathbb{V}$ is defined as

$$
\mathbb{V}(x(k)):=\left\{u(k) \in \mathbb{U} \mid A x(k)+B \triangle u(k) \in \mathbb{X}_{\mathrm{r}}\right\} .
$$

This global set is, however, applicable for the standard MPC. For MMPC, we need to consider the multiplexed inputs separately. In what follow, the subscript $(k)$ denotes the time index.

Global one-step admissible multiplexed-control set:

In a multiplexed control space, denote the ellipsoid defined by the stability constraint (11) w.r.t. subsystem control vector $u_{i}$ as $\mathbb{W}_{i}, i \in\{1,2, \ldots, m\}$.

$\triangle u_{i}(k)^{T} R_{i(k)} \triangle u_{i}(k)+2 x_{i}(k)^{T} S_{i(k)} \triangle u_{i}(k)+\psi_{i}(k) \leq 0$,

where $\psi_{i}(k):=x_{i}(k)^{T} Q_{i(k)} x_{i}(k)-\beta_{i} \xi_{i}(k-1), 0<\beta_{i}<1$, in which, $\xi_{i}(k)$ denotes $\xi\left(x_{i}(k), u_{i}(k)\right)$ of the form

$$
\xi\left(x_{i}(k), u_{i}(k)\right):=\left[\begin{array}{c}
x_{i}(k) \\
\triangle u_{i}(k)
\end{array}\right]^{T}\left[\begin{array}{cc}
Q_{i(k)} & S_{i(k)} \\
* & R_{i(k)}
\end{array}\right]\left[\begin{array}{c}
x_{i}(k) \\
\triangle u_{i}(k)
\end{array}\right] .
$$

The Cartesian product of $\mathbb{W}_{1} \times \ldots \times \mathbb{W}_{m}$ is then denoted as $\mathbb{W}$, which is an ellipsoid determined by (4) w.r.t. the global control vector $\triangle u$ having the multiplier matrices $Q, S, R$ of block diagonal type.

According to the MPC literature [16], the nominal recursive feasibility of the closed-loop $\Sigma$, subject to $x \in \mathbb{X}$, is achieved whenever $x(k) \in \mathbb{X}_{r}$ for every time step $k$. Therefore, the global recursive feasibility is obtained here by having $\mathbb{W}$ a subset of $\mathbb{V}$, assuming $x_{0} \in \mathbb{X}_{\mathrm{r}}$. The global set $\mathbb{V}$ is not applicable for MMPC, nonetheless.

For MMPC, it is possible to elaborate further with the global one-step admissible multiplexed-control set, defined next. For clarity, rewrite (7) as

$x(k+1)=A x(k)-B u(k-1)+B_{[i]} u_{i}(k)+B_{[j]} u_{[j]}(k)$,

where $u_{[j]}$ is the stacking vector of all planned control moves $u_{j}(k), j \neq i$. Denote the sum of known components as $x_{i,[j]}:=A x(k)-B u(k-1)+B_{[j]} u_{[j]}(k)$. The global onestep admissible multiplexed-control set is then defined as

$$
\mathbb{V}_{i}(x(k)):=\left\{u_{i}(k) \in \mathbb{U}_{i} \mid x_{i,[j]}+B_{[i]} u_{i}(k) \in \mathbb{X}_{\mathrm{r}}\right\}
$$

It is apparently that $\mathbb{V} \subseteq \mathbb{V}_{1} \times \ldots \times \mathbb{V}_{m}$, but not reversely, since $\mathbb{V}_{i}$ is computed from a known value of a planned control move $u_{j}, j \neq i$, but not a set of $u_{j}$. This means that the local state constraint satisfaction $\left(x_{i}(k+1) \in \mathbb{X}_{i}\right)$ at the next time step $k+1$ may not be obtained by having $u_{i}(k) \in \mathbb{V}_{i}(x(k))$ alone. It is, therefore, necessary to have the iterative feasibility condition derived in the next subsection.

Remark 2: $\mathbb{V}_{i}$ can be calculated in Matlab by the following two steps using the range function in MPT toolbox [20]:

$$
\begin{aligned}
& \mathbb{V}_{\text {temp }}:=\left\{d \in \mathbb{R}^{n} \mid H_{d} d \leqslant f_{d}\right\},(\text { dummy polytope }) \\
& =\text { range }\left(\mathbb{X}_{r}, I_{n},-A x(k)+B u(k-1)+B_{[j]} u_{[j]}(k)\right)
\end{aligned}
$$

and subsequently, $\mathbb{V}_{i}=\left\{u_{i} \in \mathbb{R}^{m_{i}} \mid H_{i} u_{i} \leqslant f_{i}\right\}$, where $H_{i}=H_{d} B_{[i]}, f_{i}=f_{d}$, in which $\left(H_{d}, f_{d}\right)$ and $\left(H_{i}, f_{i}\right)$ are the H-representation of the respective polytopes.

\section{B. Local iterative feasibility}

It is assumed that the interactive input $v_{i}(k)$ is known to the local subsystem $\mathcal{S}_{i}$. For the local state constraint satisfaction at the next time step (i.e. $x_{i}(k+1) \in \mathbb{X}_{i}$ whenever $\left.x_{i}(k) \in \mathbb{X}_{i}\right)$, it additionally requires that $u_{i}(k)$ belongs to the local one-step admissible control set of $\mathcal{S}_{i}$, taking the neighboring interactions into account. The onestep admissible control set of $\mathcal{S}_{i}$ is an invariant set of $u_{i}$, denoted as $\mathbb{B}_{i}$, such that for the known $x_{i}(k)$ and $v_{i}(k)$ and all $u_{i} \in \mathbb{B}_{i}$, we always have $x_{i}(k+1) \in \mathbb{X}_{i}$, i.e.

$$
\mathbb{B}_{i}:=\left\{u_{i}(k) \in \mathbb{U}_{i} \mid\left[A_{i} x_{i}(k)+E_{i} v_{i}(k)\right]+B_{i} \triangle u_{i}(k) \in \mathbb{X}_{i}\right\}
$$

$\mathbb{B}_{i}$ is determined by using two steps similar to those in computing $\mathbb{V}$, but for a subsystem $\mathcal{S}_{i}$.

\section{Recursive feasibility for MMPC}

For the feasibility of the multiplexed input $u_{i}(k)$, it is apparently that we need the condition of

$$
\mathbb{W}_{i} \cap \mathbb{B}_{i} \cap \mathbb{V}_{i} \neq \varnothing,
$$

and this is achievable by two separate conditions of

$$
\mathbb{W}_{i} \subseteq \mathbb{B}_{i} \neq \varnothing \text { and } \mathbb{W}_{i} \cap \mathbb{V}_{i} \neq \varnothing
$$

In this work, we obtain the condition of $\mathbb{W}_{i} \subseteq \mathbb{B}_{i}$ by re-computing the multiplier matrices $Q_{i(k)}, S_{i(k)}, R_{i(k)}$, as stated in Theorem 2 . The second condition is an assumption.

\section{Assumption 2: $\mathbb{W}_{i} \cap \mathbb{V}_{i} \neq \varnothing$.}

This assumption is reasonable, as it is inferred by the controllability of the constrained $\mathcal{S}_{i}$. In order to derive the LMI condition for $\mathbb{W}_{i} \subseteq \mathbb{B}_{i}$, we need to use an ellipsoidal $\mathbb{B}_{i}$ instead of a polytope. Denote the maximum ellipsoid inscribed in the polytope $\mathbb{B}_{i}$ as $\mathbb{E}_{i}$,

$$
\mathbb{E}_{i}:=\left\{u_{i} \mid\left(u_{i}-e_{i}\right)^{T} E_{i}\left(u_{i}-e_{i}\right) \leqslant 1\right\} .
$$

The ellipsoid $\mathbb{E}_{i}$ can be easily found from the above polytope of $H_{i} u_{i} \leqslant f_{i}$. Then, the recursive feasibility of MMPC is achieved by having $\mathbb{W}_{i} \subseteq \mathbb{E}_{i}$. 


\section{Stability condition for constrained system}

In the following theorem, the matrix $P$ of the storage function $V(x)=x^{T} P x$ is computed as in an LQR problem using Riccati equation. It is thus known in below LMI optimizations. Since the dynamic multiplier matrices $Q_{(k)}, S_{(k)}, R_{(k)}$ are used instead of the static $Q, S, R$, their initial values will be determined from the dissipative condition for the openloop system $\Sigma$.

Assume that $Q_{(1)}=\operatorname{diag}\left[Q_{i(1)}\right]_{1}^{m}, S_{(1)}=\operatorname{diag}\left[R_{i(1)}\right]_{1}^{m}$, $R_{(1)}=\operatorname{diag}\left[R_{i(1)}\right]_{1}^{m}$ are computed from solving (6) with $P$ fixed, $\xi_{i(0)}>0$.

Theorem 2: Let $0<\alpha<1 \quad 0<\beta_{i}<1, \beta=\max _{i=1}^{m} \beta_{i}$, and $\frac{\alpha}{\beta}<1$. Suppose that whenever $\xi_{(k-1)} \leq 0$, the following online LMI optimizations are feasible for $i=1 \ldots m$ :

$$
\begin{gathered}
\max _{Q_{i(k)}, S_{i(k)}, R_{i(k)}, \lambda_{i}>0} \quad x_{i(k)}^{T} Q_{i(k)} x_{i(k)} \\
\left.* \begin{array}{cc}
A^{T} P A-\alpha P-Q_{(k)} & A^{T} P B-S_{(k)} \\
* & B^{T} P B-R_{(k)}
\end{array}\right] \prec 0 \\
\text { where } Q_{(k)}=\operatorname{diag}\left[Q_{j(k)}\right]_{1}^{m}, R_{(k)}=\operatorname{diag}\left[R_{j(k)}\right]_{1}^{m}, \\
S_{(k)}=\operatorname{diag}\left[S_{j(k)}\right]_{1}^{m}, \text { in which } \\
Q_{j(k)}=Q_{j(k-1)}, S_{j(k)}=S_{j(k-1)}, R_{j(k)}=R_{j(k-1)} \\
\text { if } j \neq i, j \in\{1,2, \ldots, m\}, \text { and } \\
\psi_{i(k)}^{T}=x_{i(k)}^{T} Q_{i(k)} x_{i(k)}-\beta_{i} \xi_{i(k-1)}
\end{gathered}
$$

Then any $m$ multiplexed controls $u_{i}(k) \in \mathbb{W}_{i}$ that employ the resultant $Q_{i(k)}, S_{i(k)}, R_{i(k)}, i=1 \ldots m$, asymptotically stabilize the constrained $\Sigma$, and further, $\mathbb{W}_{i} \subseteq \mathbb{B}_{i}$.

Proof: The proof is similar to those in [10], plus the condition for $\mathbb{W}_{i} \subseteq \mathbb{E}_{i}$ which is cast in LMI in (18). Refer to Appendix A for the full proof.

On the ground of this stability condition, the MMPC optimization with recursive feasibility and stability constraints are as follows:

$$
\begin{array}{r}
\min \mathcal{J}_{k}=\sum_{\ell=0}^{N-1}\left\|x_{k+\ell \mid k}^{T}\right\|_{\mathcal{X}}^{2}+\left\|\Delta \tilde{u}_{k+\ell \mid k}^{T}\right\|_{\mathcal{U}}^{2}, \quad(19 \mathrm{a}) \\
\text { w.r.t. } \triangle \tilde{u}_{k+\ell \mid k}, \quad(\ell=0, m, 2 m, \ldots, N-1) \\
\text { s.t. } \triangle \tilde{u}_{k+\ell \mid k}+u_{\sigma(k+\ell) \mid k-1} \in \mathbb{U}_{\sigma(k+\ell)},(\ell=0, m, \ldots, N-1) \\
\triangle \tilde{u}_{k+\ell \mid k}+u_{\sigma(k+\ell) \mid k-1} \in \mathbb{V}_{\sigma(k+\ell)},(\ell=0, m, \ldots, N-1) \\
\tilde{x}_{k+\ell \mid k} \in \mathbb{X},(\ell=1, \ldots, N-1) \\
x_{k+\ell+1 \mid k}=A x_{k+\ell \mid k}+B_{\sigma(k+\ell)} \triangle \tilde{u}_{k+\ell \mid k}, \quad(19 \mathrm{~d}) \\
\triangle \tilde{u}_{k+\ell \mid k}=\triangle \tilde{u}_{k+\ell \mid k-1},(\ell \neq 0, m, 2 m, \ldots, N-1), \quad(19 \mathrm{~g}) \\
\triangle \tilde{u}_{k \mid k}^{T} R_{(k)} \triangle \tilde{u}_{k \mid k}+2 x_{k \mid k}^{T} S_{(k)} \triangle \tilde{u}_{k \mid k}+\psi_{(k)} \leq 0 .
\end{array}
$$

Only one constraint (19d), which represents the local iterative feasibility condition of $u_{i} \in \mathbb{V}_{i}$, has been added to the MMPC optimization problem (8). The stability constraint (19h) is the implementation of $u_{i} \in \mathbb{W}_{i}$. It is feasible (as $\mathbb{W}_{i} \subseteq \mathbb{E}_{i} \subseteq \mathbb{B}_{i}$ ) by using the multiplier matrices provided from Theorem 2. The MMPC algorithm is now summarized below.

Algorithm 2 (MMPC with QDC-based stability constraint and feasibility constraint)

Initialization: Determine $\mathbb{X}_{\mathrm{f}}$, then $\mathbb{X}_{\mathrm{r}}$. Set $k \equiv 0$ and $\xi_{(0)}>0$. Compute $P$, then $Q_{(1)}, S_{(1)}, R_{(1)}$ by solving (6). Solve (19) over all decision variables $\triangle \tilde{u}_{k+\ell \mid k}$, for $\ell=0,1, \ldots, N-1$, respecting only constraints (19c) and (19e)-(19f).

1) Apply control move $\triangle u_{\sigma(k), k}=\triangle \tilde{u}_{k \mid k}$.

2) Store planned moves $\triangle \tilde{u}_{k+j \mid k}, j=m, 2 m, \ldots$.

3) Pause for one time step, increment $k$, obtain new measurement $x_{k}$.

4) Find $\mathbb{V}_{i}$ and $\mathbb{E}_{i}$.

5) Solve (16) for $Q_{(k)}, S_{(k)}, R_{(k)}$.

6) Solve (19) for $\tilde{u}_{k}$, respecting all constraints.

7) Go to step 1 .

\section{E. Computational Efficiency}

Thanks to MMPC, the computational time is reduced in two ways in the above algorithm.

- The vector size for computing the one-step admissible control set $\mathbb{V}$ is now reduced to $m_{i}$ from $m_{i} \times m$, corresponding to the index number $\sigma(k+\ell)$ of the multiplexed-step in the MMPC. The computational burden for the set operation is thus reduced.

- Similarly, the QDC-based stability constraint at each multiplexed-step is now a constraint w.r.t $u_{i}$ of size $m_{i}$, corresponding to the index number $\sigma(k+\ell)$ of the multiplexed-step in the MMPC, instead of $u$ of size $m_{i} \times m$. Further, this constraint is on the current control vector only $u_{i}(k)$, thus requires much less computations than the constraint on the global state vector at the end of the predictive horizon, $x(k+N) \in \mathbb{X}_{\mathrm{f}}$.

- For $u_{i}$ having only one element, i.e. a scalar variable, the computation is further simplified since $\mathbb{E}_{i} \equiv \mathbb{B}_{i}$, and is a line segment. The computational cost of finding $\mathbb{V}_{i}, \mathbb{B}_{i}$ and the matrices $Q, S, R$ will be reduced significantly.

\section{ILLUSTRATIVE EXAMPLE}

A text-book example of Automatic Generation Control in a multi-area power system is numerically simulated in this illustrative example for MMPC. Small signal models of multi-machine power systems are used in this AGC problem [21]. In the state equations, all variables are in small-variation incremental forms. The manipulated variable is the electrical output power reference $\Delta P_{\text {ref }_{i}}$ of the local generator. The state vector consist of angular frequency $\Delta \omega_{i}$, mechanical power $\Delta P_{\text {mech }_{i}}$, steam valve position $\Delta Z_{\mathrm{v}_{i}}$, and the total tie-line power $\Delta P_{\mathrm{tie}_{i}}$. The coupling variables are the negative angular frequency $-\Delta \omega_{\ell}$ and tie-line 
power $\Delta P_{\mathrm{tie}_{i \ell}}$ of all $\nu_{i}$ respective neighbours. The power flowing across a transmission line is modeled using the DC load flow (i.e. linear) method shown in Chapter 6 of [21].

Electro-mechanical state equations:

$$
\begin{aligned}
& \frac{d \Delta \omega_{i}}{d t}+\frac{1}{M_{i}} D_{i} \Delta \omega_{i}+\frac{1}{M_{i}} \Delta P_{\mathrm{tie}_{i}}-\frac{1}{M_{i}} \Delta P_{\mathrm{mech}_{i}}=-\frac{1}{M_{i}} \Delta P_{\mathrm{L}_{i}}, \\
& \frac{d \Delta P_{\operatorname{mech}_{i}}}{d t}+\frac{1}{T_{\mathrm{ch}_{i}}} \Delta P_{\mathrm{mech}_{i}}-\frac{1}{T_{\mathrm{ch}_{i}}} \Delta Z_{\mathrm{v}_{i}}=0, \\
& \frac{d \Delta Z_{\mathrm{v}_{i}}}{d t}+\frac{1}{T_{\mathrm{G}_{i}}} \Delta Z_{\mathrm{v}_{i}}-\frac{1}{T_{\mathrm{G}_{i}}} \Delta P_{\mathrm{ref}_{i}}+\frac{1}{R_{i} T_{\mathrm{G}_{i}}} \Delta \omega_{i}=0, \\
& \frac{d \Delta P_{\mathrm{tie}_{i}}}{d t}=\sum_{\ell \in\left\{\nu_{i}\right\}} T_{\mathrm{tie}_{\mathrm{i}} \ell}\left(\Delta \omega_{i}-\Delta \omega_{\ell}\right), \\
& \text { for } \nu_{i} \text { neighbors, } \nu_{i}=\nu_{i_{\mathrm{in}}}+\nu_{i_{\mathrm{out}}}, \\
& \Delta P_{\mathrm{tie}_{i}}=\sum_{\ell=1}^{\nu_{i}} \Delta P_{\mathrm{tie}_{i \ell}}, \Delta P_{\mathrm{tie}_{i \ell}}=-\Delta P_{\mathrm{tie}_{\ell i}}, \frac{d \Delta P_{\mathrm{L}_{\mathrm{i}}}}{d t}=0 .
\end{aligned}
$$

Nomenclature:

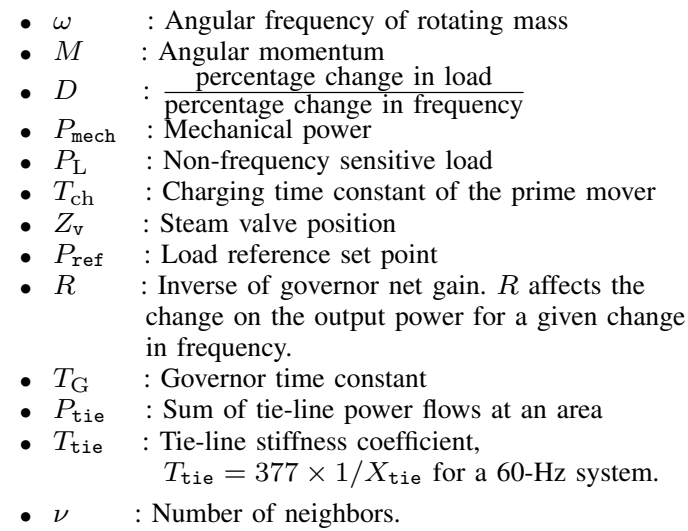

The state space model is then obtained by using parameter values in [22], [23]. The control constraints of $\eta_{i}=0.5, i=$ $1,2,3,4$ are imposed on respective subsystems. The weighting matrices of $\mathcal{X}_{1}=\operatorname{diag}\{1,0.1,0.1\}, \mathcal{X}_{2}=\mathcal{X}_{3}=\mathcal{X}_{4}=$ $\operatorname{diag}\{10,10,0.1,0.1\}, \mathcal{U}_{1}=\mathcal{U}_{2}=\mathcal{U}_{3}=\mathcal{U}_{4}==\operatorname{diag}\{1\}$ are chosen. The QDC coefficients $\beta_{1}=\beta_{2}=\beta_{3}=\beta_{4}=0.9999$ are deployed. The control and state trends from the numerical simulations in Matlab are provided in Figure 4, showing a stabilizability of MMPC with QDC-based stability constraint. The control performance here approximately matches those from a traditional MPC via QDC in previous work. The process of computing $\mathbb{V}_{i}, \mathbb{B}_{i}, \mathbb{E}_{i}$ is shortened by around six seconds (Matlab running on PC with Intel Xeon CPU 3.2 GHz, 16.0 GB RAM) for a sixty step simulation, or approximately 0.1 seconds for every computational step, compared with 0.5 seconds per step when standard MPC is used instead of MMPC, namely a saving of about $20 \%$, conservatively.

\section{CONCLUSION}

An MMPC scheme for interconnected systems has been presented. The Quadratic Dissipativity Constraint (QDC) is been employed in the input-and-power-to-state stability condition for the global system. The one-step admissible control sets are derived from the maximal output admissible set for the global system via set operations. The recursive

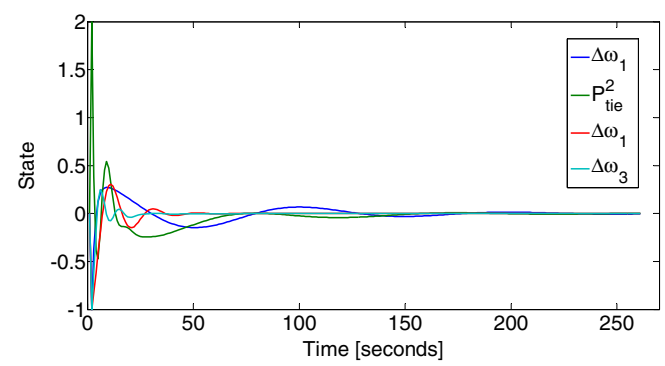

(a) State Trend.

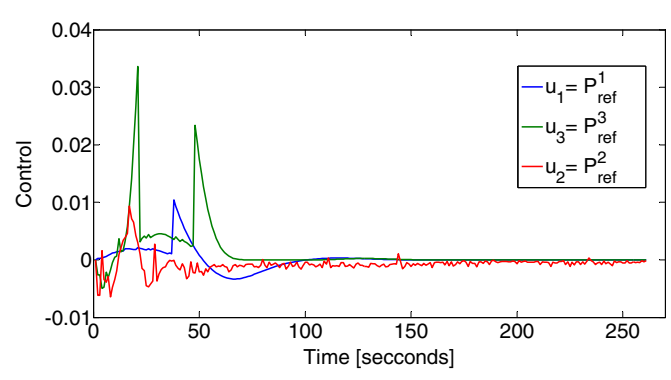

(b) Control Input Trend.

Fig. 4. AGC of a Four-Area Power Systems with MMPC and QDC-based Stability Constraint.

feasibility to hard constraints is guaranteed with the onestep admissible multiplexed-control sets applying to the MMPC optimization together with the QDC-based stability constraint. The computational cost of MMPC is reduced thanks to the QDC-based stability constraint, while that of the set operation is reduced by employing the multiplexed inputs of MMPC.

\section{REFERENCES}

[1] Ling K-V., J. Maciejowski, and B. F. Wu, "Multiplexed model predictive control," in Proc. of $16^{\text {th }}$ IFAC World Congress, (Prague, Czech Republic), 2005.

[2] Ling K-V., J. Maciejowski, A. Richards, and B. F. Wu, "Multiplexed model predictive control," Automatica, vol. 48, pp. 396-401, 2012.

[3] Maciejowski J. M., Predictive Control With Constraints. Prentice Hall, 2002.

[4] Barrett, R., M. Berry, T. F. Chan, J. Demmel, J. Donato, J. Dongarra, V. Eijkhout, R. Pozo, C. Romine, and H. V. der Vorst, Templates for the Solution of Linear Systems: Building Blocks for Iterative Methods, 2nd Ed. SIAM, 1994.

[5] Ling K-V., W. K. Ho, B. F. Wu, A. Lo, and H. Yan, "Multiplexed MPC for multi-zone thermal processing in semiconductor manufacturing," IEEE Transactions on Control Systems Technology, vol. 18, no. 6, pp. 1371-1380, 2010.

[6] Hua, Y-L., M. Z. Q. Chen, and Z. Hou, "Multiplexed model predictive control for active vehicle suspensions," International Journal of Control, vol. 88, no. 2, pp. 347-363, 2014.

[7] Richter, H., A. Singaraju, and J. S. Litt, "Multiplexed predictive control of a large commercial turbofan engine," Journal of Guidance, Control, and Dynamics, vol. 31, no. 2, pp. 273-281, 2008.

[8] Hartley, E. N., J. Maciejowski, and K.-V. Ling, "Performance evaluation of multiplexed model predictive control for a large airliner in nominal and contingency scenarios," Proc. of IEEE American Control Conference, pp. 1199-1204, Montreal, Canada, 2012. 
[9] Ling K-V., J. Maciejowski, J. Guo, and E. Siva, "Channel-hopping model predictive control," in Proc. of $18^{\text {th }}$ IFAC World Congress, (Milano, Italy), 2011.

[10] Tri Tran, K.-V. Ling, and J. M. Maciejowski, "Model predictive control via quadratic dissipativity constraint," Proc. of the $53^{\text {rd }}$ IEEE Conference on Decison and Control, pp. 6689-6694, Los Angeles, CA, December 2014.

[11] Rakovic, S. V. and R. H. Gielen, "Positively invariant families of sets for interconnected and time-delay discrete-time systems," SIAM Journal on Control and Optimization, vol. 54, no. 4, pp. 2261-2283, 2014.

[12] Blanchini F., "Set invariants in control," Automatica, vol. 35, pp. 1747$1767,1999$.

[13] Kolmanovsky I. and E. Gilbert, "Theory and computation of disturbance invariant sets for discrete-time linear systems," Mathematical Problems in Engineering: Theory - Methods and Applications, vol. 4, pp. 317-367, 1998.

[14] Tri Tran, K.-V. Ling, and J. M. Maciejowski, "Model predictive control of nonlinear input-affine systems with feasibility and stability constraints," Proc. of the $10^{\text {th }}$ International Conference on Automation, Robotic, Control and Vision, pp. 992-997, Singapore, December 2014.

[15] Tan Z., Y. C. Soh, and L. Xie, "Dissipative control of linear discretetime systems," Automatica, vol. 35, pp. 1557-1564, 1999.

[16] Mayne D. Q., J. B. Rawlings, C. V. Rao, and P. O. M. Scokaert, "Constraint predictive control: Stability and optimality," Automatica, vol. 36, pp. 789-814, 2000.

[17] Gilbert, E.G. and K. Tan, "Linear systems with state and control constraints: The theory and application of maximal output admissible sets," IEEE Transactions on Automatic Control, vol. 36, no. 9, pp. 1008-1020, 1991.

[18] Kerrigan E. C. and J. M. Maciejowski, "Invariant sets for constrained nonlinear dicrete time systems with application to feasibility in model predictive control," Proc. of the $39^{\text {th }}$ IEEE Conference on Decision and Control, pp. 4951-4956, Sydney, Australia, 2000.

[19] Rakovic, S. V., E. C. Kerrigan, D. Q. Mayne, and K. I. Kouramas, "Optimized robust control invariance for linear discrete-time systems: Theoretical foundations," Automatica, vol. 43, pp. 831-841, 2007.

[20] Kvasnica, M, P. Grieder, M. Baotic, and F. Christophersen, "MPT Multiparametric toolbox," tech. rep., ETH-Swiss Federal Institute of Technology, Zurich, 2006.

[21] Wood, A. J., B. F. Woolenberg, and G. B. Shebl, Power Generation Operation and Control, $3^{\text {rd }}$ Edition. New Jersey: John Wiley \& Son, 2013.

[22] Tri Tran, K.-V. Ling, and J. M. Maciejowski, "Application of quadratically-constrained model predictive control in power systems," In Proc. of the $10^{\text {th }}$ International Conference on Automation, Robotic, Control and Vision, pp. 193-198, Singapore, December 2014.

[23] Tri Tran, K.-V. Ling, and J. M. Maciejowski, "Closed-loop development for quadratic dissipativity constraint," Proc. of the $3^{\text {rd }}$ International Conference on Control, Automation, and Information Science, pp. 24-29, Gwangyu, South Korea, December 2014. 